II. Kritik von Regierungstechniken in der Krise 



\title{
Mundschutz oder mundtot? \\ Corona-Krise als Ausnahmezustand für und durch die Demokratie
}

\author{
Demokrat Ramadani
}

\section{Corona-Krise als Ausnahmezustand: eine Befragung}

Kontaktverbot im öffentlichen Raum, Abriegelung der Nordseeinseln, Ausgangssperren in einigen Bundesländern, Übermittlung von Bewegungsdaten an das Robert-Koch-Institut (RKI) durch die Telekom, SchlieBung von Kindertagesstätten sowie Grundschulen, weiterführenden Schulen und Hochschulen, Maskenpflicht beim Einkaufen und im ÖPNV, Home Office oder Kurzarbeit für viele Beschäftigte, wirtschaftliche Einbußen für Unternehmen und Solo-Selbständige. Es werden viele weitere Maßnahmen für das Verhalten im Alltag empfohlen. Mancherorts versinken Menschen in Einsamkeit und Angst, andernorts finden Kneipenschlägereien und Angriffe auf Menschen statt, die in der Öffentlichkeit husten - die Liste der Konsequenzen aus der Covid-19-Epidemie (nachfolgend: Corona) ist lang. Es ist eine breite Palette von Veränderungen und Einschränkungen. Für alle handelt es sich zweifellos um eine Ausnahmesituation, viele sprechen gar von einem Ausnahmezustand. ${ }^{1}$

Ausnabmezustand ist ein politik- und rechtstheoretisches Konzept, das viel diskutiert wird. Lemke charakterisiert ihn allgemein als besondere Regierungstechnik, die eine Konzentration der Kompetenzen bei der Exekutive vorsieht (vgl. Lemke 2017: 1f.). Drei Merkmale seien kennzeichnend: Erstens bedarf es einer plausiblen Behauptung, dass eine Krise vorliegt und dass diese Krise nicht im Normalbetrieb der Verfassung bewältigt werden könne. Zweitens werden Machtbefugnisse in den Händen der Exekutive konzentriert, womit eine Verschiebung der Kompetenzen innerhalb des machtteiligen Gefüges stattfindet. Drittens werden Maßnahmen getroffen, die nicht notwendigerweise zeitlich beschränkt sind, selbst wenn die Verfassungen eine solche Temporalität vorsehen.

1 Siehe beispielhaft die Chronik zur Corona-Krise der Tagesschau (2020) oder einen Meinungskommentar der WELT (2020). 
Heute verfügen 76 der 86 laut Freedom House als qualitativ höchstwertig eingestuften Demokratien über jeweils spezifisch ausgestaltete Regelungen zum Ausnahmezustand (vgl. Lemke 2017: 4). Auch die Bundesrepublik Deutschland (BRD) gehört zu jenen Gemeinwesen, die über eine Notstandsverfassung verfügen. Als Notstandsverfassung im engen Sinne lassen sich laut Grundgesetz der äußere Notstand als Bedrohung durch ausländische militärische Gewalt und der innere Notstand als Gefahrenquellen, die innerhalb des Staatsgebietes angesiedelt sind - insbesondere Naturkatastrophen, Unglücksereignisse, Aufruhr und Bürgerkrieg - unterscheiden. Im ersten Fall gelten die Artikel 80a und 115a bis 115l GG und für den zweiten Fall gilt Artikel 35 GG (vgl. Gramm/Wittenberg 2018: Rn. 43 ff.). Zur Notstandsverfassung im weiten Sinne zähle ich die notstandsähnlichen Gesetze zur Gefahrenabwehr, worunter das Infektionsschutzgesetz (IfSG) fällt (vgl. Erdle 2020). Es ist eingebettet in das System internationaler Pandemiebekämpfung, die durch die Weltgesundheitsorganisation (WHO) verantwortet wird. Wenn diese die, Public Health Emergency of International Concern' ausruft, dann sind die Mitgliedsstaaten angehalten, die betreffenden Gesetze zum Seuchenschutz zu aktivieren (vgl. Fangerau/Labisch 2020: 18). Für diesen Fall enthält das IfSG weitreichende Befugnisse zur Verhütung ( $\$ S 16 f f$.$) und zur Bekämpfung übertragbarer Krankheiten$ ( $\mathbb{S}$ 24ff.). Das IfSG wird von den Bundesländern ausgeführt, sodass die Anordnung von Maßnahmen der Verhütung sowie der Bekämpfung übertragbarer Krankheiten im Grunde den Ländern obliegt. Zu diesem Zweck dürfen die zuständigen Länderbehörden laut $\$ 28$ Abs. 1 IfSG mit ihren Maßnahmen die Grundrechte der körperlichen Unversehrtheit, der Freiheit der Person, der Freizügigkeit, der Versammlungsfreiheit, des Briefund Postgeheimnisses und der Unverletzlichkeit der Wohnung einschränken sowie ein berufliches Tätigkeitsverbot verhängen. Für NordrheinWestfalen gilt das ,Infektionsschutz- und Befugnisgesetz' (IfSBG-NRW). $\$ 11$ IfSBG-NRW legt hier fest, dass der Landtag eine „epidemische Lage landesweiter Tragweite“ ausrufen muss, damit das Landesministerium für Gesundheit seine Zuständigkeit mittels Anordnungen nach $\mathbb{S} 12$ bis 14 IfSBG-NRW ausüben kann.

Vorliegend stellt sich die Frage, ob die Corona-Krise mehr als eine Notsituation ist. Ist sie ein Notstand im Sinne eines Ausnahmezustands? Und ist Ausnahmezustand gleich Ausnahmezustand? So unterscheidet Martin Sabrow zwischen staatlichem und gesellschaftlichem Ausnahmezustand (vgl. Sabrow 2020) und Christian Leonhardt zwischen souveränem und politischem Ausnahmezustand (vgl. Leonhardt 2017: 41ff.). Auch dieser Aufsatz nimmt eine Unterscheidung verschiedener Ausnahmezustände vor. Der Startpunkt für die nachfolgenden Überlegungen ist das Selbstverständnis 
radikaldemokratietheoretischer Befragung. Demokratie ist in dieser Lesart ein „Modus der steten kritischen Infragestellung“ (Flügel-Martinsen 2019: 577). Der befragende Charakter radikaldemokratischer Kritik ist Ausdruck von weitreichenden Ungewissheits- und Kontingenzannahmen.

Dieser Text unternimmt zwei radikaldemokratietheoretisch motivierte Suchbewegungen. Einen befragenden Zugang wählt das nächste Kapitel (II.). Hier wird erkundet, welche Verständnisse des Ausnahmezustands unterschieden werden können. Vorgeschlagen wird die Differenzierung zwischen dem Ausnahmezustand gegen die Demokratie (1.), für die Demokratie (2.) und durch die Demokratie (3.). Ein infrage stellender Zugang ist für das darauffolgende Kapitel gewählt (III.). Hier wird vertreten, dass die CoronaKrise ein doppelter Ausnahmezustand ist. So sind die getroffenen CoronaMaßnahmen nach dem Infektionsschutzgesetz als rechtlicher Ausnahmezustand für die Demokratie einzustufen. Zugleich wird die Corona-Krise ihrem Charakter nach als bio-politischer Ausnahmezustand durch die Demokratie gedeutet. Im Fazit wird betont, dass die Corona-Krise dynamisch ist. Demokratie ist das aber grundsätzlich auch. Wenn wir gesellschaftliche Auseinandersetzungen und den Streit um die Einrichtung der Welt auch in Krisenzeiten nicht scheuen, dann sind wir trotz Mundschutz nicht mundtot. $^{2}$

\section{Befragung: Was verstehen wir unter Ausnahmezustand?}

In diesem Kapitel werden drei Verständnisse von Ausnahmezustand skizziert: Der Ausnabmezustand gegen die Demokratie ist den Arbeiten Carl Schmitts nachempfunden. Der Ausnahmezustand für die Demokratie lässt sich laut Max Molly auf Überlegungen von Otto Kirchheimer zurückführen. Der Ausnabmezustand durch Demokratie kann anhand von Jacques Rancières Konzept des Politischen veranschaulicht werden.

Carl Schmitts Verständnis vom Ausnahmezustand muss im Zusammenhang seiner Begriffe des Politischen, der Souveränität und der Entscheidung betrachtet werden. Das Politische drückt sich bei Schmitt in der Entscheidung darüber aus, wer zum (öffentlichen) Freund oder Feind erklärt wird. Diese Entscheidung kann nur der Souverän fällen. Souverän ist dabei diejenige Figur, die die verfassungsmäßige Ordnung außer Kraft zu setzen vermag.

2 Ich danke Dorentina Reka und Liridone Ramadani für die coole Diskussion und die hilfreichen Impulse. 
Der Ausnahmezustand tritt ein, wenn der Souverän entscheidet, dass der Normalzustand mit seinen organisationsrechtlichen Zuständigkeiten, verfahrensrechtlichen Vorgaben und grundrechtlichen Garantien nicht mehr gilt. Der Ausnahmezustand ist zudem ein rechtlich fassungsloser $\mathrm{Zu}-$ stand, weil er sich im Vorfeld nicht regulieren lässt. Eine generelle Norm kann eine absolute Ausnahme niemals erfassen und daher auch die Entscheidung, dass ein echter Ausnahmefall gegeben ist, nicht begründen (vgl. Schmitt 2015: 13). Es kann also nach Schmitt kein wie auch immer ausgestaltetes Notstandsrecht geben, das festlegt, wann ein Ausnahmezustand vorliegt und was dann erlaubt ist. Während aus heutiger rechtsstaatlicher Sicht betrachtet diese Herbeiführung des Ausnahmezustandes keine Kompetenz de jure, sondern de facto ist - der Souverän also nicht so handelt, weil er es darf, sondern weil er es kann - fallen für Schmitt in der Figur des Souveräns juridische und faktische Macht ununterscheidbar zusammen. Um kenntlich zu machen, wer der Souverän ist, der zu all dem befähigt ist, muss offengelegt werden, dass das Vorbild des Ausnahmezustandes Schmittscher Lesart in der römischen Antike liegt. Dort wurde durch den Senat für einen Zeitraum von sechs Monaten das im Normalzustand nicht existente Amt des Diktators geschaffen. In ihm sind alle für die Abwehr der Krise erforderlichen Kompetenzen gebündelt und die ansonsten zuständigen Institutionen suspendiert worden (vgl. Lemke 2017: 3). Aufgegriffen wurde diese Konzeption von Machiavelli, der überzeugt davon war, dass jene Republiken zugrunde gehen werden, welche im Falle äußerster Gefahr nicht zum Mittel der Diktatur greifen (vgl. ebd.: 4). Schmitt bezieht sich auf Bodin, dessen wissenschaftlicher Erfolg ihm zufolge darin liegt, die souveräne Entscheidungsgewalt und die Ungebundenheit des Souveräns zusammengedacht zu haben (vgl. Schmitt 2015: 15).

Aus dieser Traditionslinie ergibt sich schließlich, dass der Souverän eine autoritär-präsidentiale Figur ist (vgl. Molly 2017: 73). Als kommissarischer Diktator entscheidet er darüber, wann Recht gilt und wann nicht, sowie für wen Recht gilt und für wen nicht (vgl. Lemke 2017: 5). Molly charakterisiert den Schmittschen Ausnahmezustand daher als „brachialen Zustand, als faschistische Ermächtigung" (Molly 2017: 72). Dies wird vor allem in Zusammengang mit dem Hinweis von Franz L. Neumann deutlich, dass Schmitt den Nazis nahestand, so dass seine Theorien unzweifelhaft als „Mosaikstein der NS-Ideologie“ (zitiert nach Molly 2017: 72) verstanden werden können. Schmitt sympathisierte also nicht nur mit einem Führerkult, sondern bereitete als Kronjurist der Nazis die rechtstheoretische Grundlage für eine Führerherrschaft als Ausnahmezustand gegen die Demokratie. 
Der Ausnabmezustand für die Demokratie lässt sich an Gedanken von Otto Kirchheimer andocken, der bei John Locke ansetzt (vgl. Molly 2017: 71ff.). Bei ihm werden, anders als in der römischen Antike oder bei Machiavelli, keine besonderen Institutionen geschaffen, sondern die bestehenden Institutionen mit besonderen Rechten und Kompetenzen ausgestattet. Diese beinhalten, den Ausnahmezustand auszurufen, Maßnahmen zu seiner Bewältigung zu treffen (worunter auch die Einschränkung bestimmter Rechte fallen kann) und ihn zu beenden (vgl. Lemke 2017: 3). Der Ausnahmezustand für die Demokratie zeichnet sich also dadurch aus, dass er demokratisch legitimiert, konstituiert und fundiert ist: Der Eintritt ist demokratisch legitimiert, weil jene Organe darüber befinden, die sich dafür vor dem Demos rechtfertigen müssen und letztlich das Risiko tragen, für diese Entscheidung abgestraft zu werden. Der Ausnahmezustand ist demokratisch konstituiert, weil das Notstandsrecht nicht die Demokratie außer Kraft setzt, sondern eine Version von Demokratie schafft, die der effektiven Gefahrenabwehr dient. Es ist klar geregelt, wann und von wem er ausgerufen werden darf, wer welche Zuständigkeit währenddessen hat, welche Maßnahmen ergriffen werden können und wann der Ausnahmezustand als beendet gilt. Schließlich ist der Ausnahmezustand demokratisch fundiert. Es gibt Schutzmechanismen, die die Demokratie vor der Instrumentalisierung des Ausnahmezustands bewahren. Es bleiben alle demokratischen Vorkehrungen von checks and balances bestehen. Ein demokratischer Ausnahmezustand ist demnach kein brachialer Einbruch einer neuen, autoritären Ordnung (vgl. Molly 2017: 82). Der Umstand, dass die Ausnahme keine Ausnahme von der Demokratie, sondern eine Ausnahme in der und für die Demokratie ist, wird auch daran deutlich, dass die Menschen nicht plötzlich zu Objekten eines obrigkeitsstaatlichen Ausnahmeregenten werden. Der Kern eines demokratischen Ausnahmezustandes liegt somit in den von Kirchheimer bemerkten Auswirkungen auf die Einzelnen im System: Staatliche, politische und rechtliche Praktiken können demnach im Versuch stehen, Meinungen und Handlungen, Individuen und Gruppen in der Ausgestaltung des Ausnahmezustandes einzubeziehen oder auszuschließen (vgl. ebd.: 71) - je nachdem, was für die Gefahrenabwehr gerade nützlich ist. Niemandes Position wird jedoch annihiliert.

Der Ausnahmezustand durch die Demokratie lässt sich am besten anhand von Jacques Rancières Politikkonzept verdeutlichen. Seine Unterscheidung zwischen der Polizei und der Politik erinnert an Claude Leforts Differenzierung zwischen der Politik und dem Politischen.

Als Polizei versteht er die Institutionen, Strukturen und Normen, die eine gesellschaftliche Ordnung konstituieren. Ausgangspunkt für Rancière ist die Feststellung, dass jede Gesellschaftsordnung durch eine Machtver- 
teilung über das Sichtbare, Sagbare und Wahrnehmbare gekennzeichnet ist (vgl. Abbas 2019: 390). Es gibt keine Art von menschlichem Zusammensein ohne Polizei (vgl. Leonhardt 2017: 47). Als hierarchische Gesellschafts- und Herrschaftsordnung umfasst sie zum einen die staatlichen Institutionen und Prozesse, durch die Gemeinschaften regiert werden. Zum anderen fallen darunter jene Verfahren, anhand derer Menschen Plätze und damit zusammenhängende soziale Funktionen, Identitäten, Fähigkeiten und Tätigkeiten zugewiesen werden (vgl. Abbas 2019: 391). Diese Ordnung schließt immer auf bestimmte Weise einen Teil der Menschen ein (diese gelten als gleichberechtigte Subjekte) und einen anderen Teil aus (das sind die Anteillosen). Während die Polizei also ein Modus der Ungleich-Machung ist, ist die Politik demgegenüber ein Modus der GleichSetzung.

Politik ist bei Rancière jenes Phänomen oder jener Prozess, der polizeiliche Ordnung infragestellt. Das Wesentliche der Politik ist der Streit über die Konstitution der Polizei und damit über „die Frage nach dem, was sichtbar und was sagbar ist" (Leonhardt 2017: 49). Politik erschüttert die polizeiliche Ordnung, indem sie eine Einbindung jener Ausgeschlossenen unter Berufung auf Freiheit und Gleichheit fordert (vgl. Abbas 2019: 392). Politik ist für Rancière das Handeln von Menschen, denen es durch die Austragung eines Dissenses gelingt, dass ihr Sprechen gehört und nicht bloß als Lärm abgetan wird. Subjekte, Positionen und Themen werden entgegen der polizeilichen Zuschreibung als relevant erkämpft (vgl. ebd.: 393). Kurzgefasst: Das Wesen der Politik ist ein Streit darüber, wer auf welche Weise gehört und einbezogen werden soll, welche Themen zur Sprache kommen und welche Fragen als politisch relevant eingestuft werden.

Weil die meisten heutigen Demokratien in Rancières Augen aber einen solchen Dissens nicht mehr praktizieren, sind sie für ihn Post-Demokratien. Die Gefahr für die Demokratie sieht Rancière deshalb im vorherrschenden polizeilichen Regierungsstil des Konsenses. Der Konsens macht jede Diskussion überflüssig. Wenn überhaupt, dann kommt sie nur noch als Schein vor. Der Konsens negiert den Streit nicht, indem er ihn einfach ignoriert, sondern indem er eine vollständige Sichtbarkeit und Einbeziehung aller vorgibt (vgl. Leonhardt 2017: 51). Er ist eine Entpolitisierungsmaschine, eine „Reduktion der Politik auf die Polizei“ (ebd.: 52). Dabei ist die Unterbrechung der Ordnung durch den Streit das Momentum der Demokratie schlechthin (vgl. ebd.: 47). Da dieser ihr verloren gegangen ist, handelt es sich gleichsam um eine eingeschlafene Demokratie.

Der Ausnahmezustand durch die Demokratie ist nun eine Wiedererweckung der Demokratie aus sich selbst heraus. Er demonstriert, dass das polizeiliche System und der vorherrschende Modus des Konsenses gestört 
werden können und müssen. Störung ist hier nicht unbedingt zu verstehen als Lähmen, Bremsen, Ver- oder Behindern, sondern als emanzipativer Akt des Aufbrechens. Der Ausnahmezustand durch Demokratie ist eine Infragestellung der Souveränität, indem immer wieder neu über die Frage des Sprechens und der Ordnung gestritten wird (vgl. Leonhardt 2017: 46). Das Demokratische besteht hier also in der „Einrichtung einer Bühne des Dissenses“, der Einrichtung des Konfliktes über „das Dasein einer gemeinsamen Bühne“ sowie über "das Dasein und die Eigenschaft derer, die auf [der Bühne; D.R.] gegenwärtig sind“ (Leonhardt 2017: 50).

\section{Infragestellung: Corona-Krise als doppelter Ausnahmezustand?}

Eine schwere Krankheit, so heißt es, offenbart den Charakter eines Menschen, eine Epidemie offenbart den Charakter einer Gesellschaft (vgl. Fangerau/Labisch 2020: 127). Wie ist es also um unsere Gesellschaft bestellt in Zeiten von Corona? Eine pauschale Antwort lässt sich nicht geben. Der vorliegende Aufsatz bemüht das Bild des doppelten Ausnahmezustandes: Die Corona-Maßnahmen sind einerseits ein infektionsrechtlicher Ausnahmezustand für die Demokratie, weil sie die Pandemie auf demokratischem Wege bekämpfen. Andererseits entstehen etliche Gegenbewegungen als kritische Resonanz auf diese Maßnahmen. Nicht alle verfolgen dabei demokratische Ziele. Jene, auf die das zutrifft (dazu gleich ausführlicher), können einen Ausnabmezustand durch die Demokratie herbeiführen. Sie haben aus radikaldemokratietheoretischer Perspektive das Potential, mit ihrer kritischen Praxis über bestehende normative und institutionelle Verhältnisse hinauszudenken und damit zur Weiterentwicklung der Demokratie beizutragen. Sie bringen zum Ausdruck, dass „Be-Gründungen zur Disposition stehen, konkurrieren und umkämpft sind“ (Sack 2019: 506) und schaffen durch diese Deutungskonflikte politische Räume und Kommunikationsprozesse über die Grundlagen, Inhalte, Versäumnisse und anstehenden Entscheidungen der Politik (vgl. ebd.: 508). Nur wenn es „demokratische Interventionen gegen bestehende Ordnungen“ gibt (FlügelMartinsen 2019: 581), kann die Corona-Krise zum Ausnahmezustand durch die Demokratie gewendet werden.

Erste Voraussetzung dafür ist, dass die Corona-Krise überhaupt als beeinflussbar und gestaltbar angesehen wird. Jonas Heller beschreibt in seinem Beitrag ,Ausnahmezustand“ für das Video-Glossar ,Kritische Theorien in der Pandemie', dass die Corona-Krise als unpolitischer Ausnahmezustand wahrgenommen wird (vgl. Heller 2020). Weil es sich um eine Pandemie handelt, gelte zum einen die Ursache der Krise nicht als politisch, son- 
dern als natürlich bzw. biologisch. Zum anderen würden die ergriffenen Maßnahmen nicht als politische Maßnahmen aufgefasst, weil ihr Charakter vordergründig technisch-medizinischer Art sei - damit handle es sich mehr um Maßnahmen der Naturbeherrschung als um eine politische Gestaltung der Welt (vgl. ebd.). Heller legt sehr gut dar, dass Corona ein biopolitischer Ausnahmezustand ist: Es wird nämlich auf politische Weise versucht, die kollektive Gesundheit zu regulieren, indem z.B. festgelegt wird, bei welchem Infektionsniveau welche gesundheitspolitischen und polizeirechtlichen Maßnahmen zu vollziehen sind (vgl. ebd.). Auch Heiner Fangerau und Alfons Labisch ordnen die Corona-Maßnahmen als Biopolitik im Sinne Michel Foucaults Analyse politischer Zugriffe auf den Körper und das Leben ein. Mit Bezug auf Lepra, Pest und Pocken skizzierte Foucault drei unterschiedliche Formen biopolitischen Regierens (vgl. Fangerau/Labisch 2020: 109): Der Umgang mit der Lepra sah vor, dass nur die Kranken ausgeschlossen bzw. eingesperrt werden. Bei der Pest wurde versucht, größere Gruppen zu disziplinieren, vor allem durch Überwachung und Kontrolle an Grenzen und Stadtmauern sowie durch die Einschränkung des Bewegungsraumes. Bei den Pocken war es so, dass der Staat einerseits die Ausbreitung statistisch beobachtend überwachte, während er andererseits Gebrauch von Schutzimpfungen machte. Der aktuelle Umgang mit dem Corona-Virus weist große Ähnlichkeiten zu den Reaktionen auf die Pocken auf. ${ }^{3}$

Zwar werden in allen Gesellschaften Leben und Leib der Menschen öffentlich gestaltet. Von autoritären Praktiken unterscheiden sich die Praktiken demokratischer Gesellschaften aber dadurch, dass sie nur wenig bis gar keinen Gebrauch von invasiver Kontrolle und der Ausübung von Gewalt machen. Hier kontrollieren sich die Bürger"innen - gerade mit den vielfältigen Möglichkeiten durch die zunehmende Digitalisierung - zunächst einmal selbst und dann gegenseitig:

„Wenn privates Wollen und öffentliche Interessen einhergehen, wird sich die Biomacht vollständig verwirklichen: Essen, Trinken, Gewicht, Aussehen, Fitness und nun im Falle der Pandemie Kontakt- und Bewegungsdaten bieten Paradebeispiele für diese ,Selbst'-Disziplinierung [...] - das ist subtile Biopolitik in der IT-Kultur." (Fangerau/Labisch 2020: 104)

3 Für eine Reflexion der Verwendung des Biopolitikbegriffs in Bezug auf die Corona-Krise und eine Differenzierung von Regierungsrationalitäten unterschiedlicher Maßnahmen, siehe den Beitrag von Simon Duncker in diesem Band. 
Dieses Phänomen ist Teil einer Entwicklung, die als „Medikalisierung“ (ebd.: 106) bezeichnet wird und die beinhaltet, dass medizinische Deutungsmuster in vorher nicht medizinisch verstandene Lebensbereiche übertragen werden. Teil dessen ist, dass der Startpunkt einer medizinischen Intervention in den vergangenen hundert Jahren zusehends von der Erkrankung hin zum bloßen Gesundheitsrisiko verschoben wurde.

„Die Medizin der Moderne übernimmt über den Gesundheitsbegriff das Definitionsmonopol und über den Krankheitsbegriff das Handlungsmonopol über die individuellen und öffentlichen Körper der Menschen.“(Ebd.: 111)

Die jeweilige Deutung von Gesundheit vermittelt zugleich zwischen der Gesellschaftlichkeit und der Natürlichkeit der Menschen und ordnet so die biologischen und sozialen Grundlagen menschlichen Daseins (vgl. ebd.: 114).

Maßnahmen öffentlicher Gesundheitssicherung werden so in einem von Macht und Herrschaft sowie von pluralen Sinn- und Wertsetzungen durchtränkten öffentlichen Raum definiert und durchgesetzt (vgl. Fangerau/Labisch 2020: 117). In offenen Gesellschaften kann hier der Raum für öffentlichen Diskurs über Krankheiten und Epidemien, über Gesundheitsverhalten, Prävention, Risiko und Abwägung entstehen. Dieser Diskurs ist zwingend notwendig, um die Bedeutung von Vorgängen, die durch öffentliche Gesundheitssicherung hervorgerufen werden, zu klären, ihre Wertigkeiten zu bestimmen und Maßnahmen der Gesundheitssicherung zu bewerten (vgl. ebd.: 117). Auf die Frage nach der Biologie und der humanpathogenen Wirkung eines Erregers folgen schnell gesellschaftliche Fragen (vgl. ebd.: 128): Was ist ein Menschenleben wert? Welche Rolle spielt es bei der Bewertung, ob jemand alt, jung, gesund oder krank ist? Welche Infrastruktur halten wir vor? Was lassen wir uns eine permanente öffentliche Gesundheitssicherung kosten? Wie viel Eingriffe in die individuelle Freiheit erträgt eine Demokratie?

Yves Winter betont in diesem Zusammenhang sehr zutreffend, dass die Debatten, die derzeit über die Corona-Krise geführt werden, entweder geprägt sind von einer gesundheits- oder einer wirtschaftspolitischen Logik (vgl. Winter 2020). Dabei zeigen die Corona-Maßnahmen und die verschiedenen Reaktionen darauf, dass es auf ganz grundsätzliche Abwägungen ankommt, die im Sinne einer „ongoing democratic debate“ (ebd.) bestritten werden müssen. Es braucht also eine Auseinandersetzung darüber, wie das Gesundheitssystem krisenfest ausgestattet und wie zwischen folgenden Rechtsgütern abgewogen werden soll: 1) dem Recht auf Leben und körperlicher Unversehrtheit, 2) den wirtschaftlichen Rechten auf Ei- 
gentums- und Gewerbefreiheit, 3) den politischen Rechten auf Handlungs-, Meinungs-, Versammlungsfreiheit sowie 4) den sozialen Rechten auf Bildung und Kulturgüter.

Vor allem müssen wir uns fragen, was wir unter gesellschaftlicher Normalität verstehen wollen. Sabrow weist nämlich darauf hin, dass historisch gesehen Gesellschaften Ausnahmezustände dadurch überwinden, dass sie sie durch ihre Entscheidungen widerstrebend oder emphatisch zur Normalität werden lassen (vgl. Sabrow 2020). Die Gesellschaft ist mit Michael Th. Greven gesprochen „radikal dezisionistisch[...]“, weil „,alles entscheidbar geworden [ist], für alles kann die Politik ihre Zuständigkeit erklären““ (zitiert nach Sack 2019: 507). Gleichzeitig lässt sich feststellen, dass öffentliche Politik diese Kontingenz der Entscheidung immer wieder dadurch verschleiert, dass sie eine wie auch immer begründete Alternativlosigkeit behauptet (vgl. Sack 2019: 507). Es gilt daher, einen „demokratischen Dezisionismus" (ebd.: 508) wiederherzustellen und einen Fokus darauf zu richten, dass die Welt gerade in Zeiten der Pandemie gestaltbar ist, weil wir den biologischen Ereignissen nicht voll und ganz ausgeliefert sind und den daraus folgenden gesellschaftlichen Reaktionen nicht unterworfen sein müssen.

\section{Irgendwie kein Schluss: Corona als Ausnahmezustand-und jetzt?}

Dieser Aufsatz versteht sich als radikaldemokratietheoretisch motivierter Versuch, die Corona-Krise als Ausnahmezustand zu deuten. Dazu sind zwei fragende Suchbewegungen gewählt worden: Im ersten Schritt fand ein befragender Zugang statt, um drei Typen des Ausnahmezustandes herauszuarbeiten (II.): der Ausnahmezustand gegen die Demokratie als Überführung einer demokratischen Ordnung in eine Diktatur, der Ausnabmezustand für die Demokratie als temporäre Modifikation demokratischer Institutionen und der Ausnabmezustand durch die Demokratie als Revitalisierung der Demokratie aus sich selbst heraus. Im darauffolgenden Kapitel ist ein infrage stellender Zugang gewählt worden, um zu ermitteln, inwiefern die Corona-Krise als ein doppelter Ausnahmezustand verstanden werden kann (III.). Hier sind die Corona-Maßnahmen als infektionsrechtlicher Ausnabmezustand für die Demokratie und die Corona-Krise als bio-politischer Ausnabmezustand durch die Demokratie charakterisiert worden.

Und jetzt? Was ist durch diesen Text gewonnen? Das Corona-Virus ist dynamisch. Die dadurch ausgelöste Krankheit wird noch nicht vollständig verstanden. Die gesellschaftlichen Folgen sind nicht absehbar. Die CoronaKrise hat gesundheitspolitische, bildungspolitische, wirtschaftspolitische, 
kulturpolitische, familienpolitische, freizeitpolitische und grundrechtspolitische Auswirkungen. Eine kritische Betrachtung zur Förderung eines demokratischen Empowerments müsste auf all diesen Ebenen ansetzen. Dies kann ein Aufsatz allein nicht leisten. Das Video-Glossar ,Kritische Theorien in der Pandemie', der umfangreiche ,Corona Monitor ${ }^{64}$ sowie der vorliegende Sammelband und ähnliche Publikationen sind wichtige Unternehmungen in diese Richtung. Bei all diesen Deutungen ist die Rolle des Streitens wichtig. Der Dissens ist entscheidende Voraussetzung radikaldemokratischer Politik (vgl. Vasilache 2019: 491). So gilt nicht etwa die Konflikthaftigkeit und Umkämpftheit des Politischen als die wesentliche Gefahr für Demokratie und Emanzipation, sondern die Bestrebung der endgültigen Überwindung aller Widersprüchlichkeit und Antagonismen (vgl. ebd.). Demokratie als Gesellschaftsform lebt stets von der Möglichkeit der Dissensartikulation; Sie wird durch ein Stillstellen aller Konfliktdimensionen ihres politischen wie demokratischen Charakters beraubt (vgl. ebd.: 496).

Die kritische Deutung der Corona-Krise muss demnach ihren Ausgangspunkt bei jenen Stimmen nehmen, die die Corona-Maßnahmen als Gefährdung der Demokratie einstufen. Diese Maßnahmen, so wird argumentiert, greifen in zentrale Grundrechte wie die Versammlungsfreiheit ein. Yves Winter weist in seinem Beitrag, Democracy' für das Video-Glossar ,Kritische Theorien in der Pandemie“ beispielsweise darauf hin, dass europaweit legitime Proteste gegen die unmenschliche Unterbringung von Geflüchteten auf den griechischen Inseln selbst dann kriminalisiert werden, wenn sie kein epidemiologisches Risiko darstellen (vgl. Winter 2020). Andere halten dem entgegen, dass die Corona-Maßnahmen verhältnismäßige Interventionen zum Schutz der Menschen sind (vgl. Katzenberger 2020). Der planmäßige Missbrauch des Notstandsrechts durch die Nationalsozialisten ab 1933 habe die bundesdeutsche Diskussion um ein grundgesetzliches Notstandsrecht geprägt, sodass unsere Gesellschaft für das Problem der Instrumentalisierung sensibilisiert sei (vgl. Gramm/Wittenberg 2018: Rn. 12ff.). Darüber hinaus gibt es noch etliche Stimmen, die als Verschwörungstheorien bezeichnet werden müssen. So ist zu hören und zu lesen, dass der Ausbruch des Virus von China als Coup gegen die Weltwirtschaft eingesetzt wurde oder dass die Funkmaster der 5G-Technik die Quelle des

4 Der ,Corona Monitor' dokumentiert Ereignismeldungen über politische Umwälzungen. Die Aktiven aus drei Netzwerken (Assoziation für kritische Gesellschaftsforschung, Institut für Protest- und Bewegungsforschung, AK Kritische Geographie) wollen die Grundlage für Forschung und demokratische Kontrolle schaffen. 
Virus seien (vgl. Neft 2020). Große Aufmerksamkeit erhielten die von Rechtsextremisten unterwanderten ,Hygiene-Demos', die sich als außerparlamentarische Opposition inszenieren. Sie suchen dabei bewusst Analogien zu der Protestbewegung herzustellen, die es ab 1968 gegen die Einführung der Notstandsparagraphen gab. Die Frage, wie sich demokratieförderlicher Protest eindeutig von verschwörungstheoretischem oder rechtsgesinntem Aufruhr abgrenzen lässt, kann abstrakt nur schwer beantwortet werden. Indizien für das Bestehen einer demokratisierenden Bewegung sind gegeben, wenn Dissens ausgetragen wird, um die Gesellschaft zu öffnen statt abzuschließen, die Vielfalt zu stärken statt zu bekämpfen, Menschen einzubeziehen statt auszugrenzen, Privilegien abzubauen statt zu konservieren, individuelle und kollektive Selbstbestimmung auszubauen statt einzuschränken - und wenn bei alle dem die eigene Fehlbarkeit nicht geleugnet, sondern anerkannt wird.

Radikale Demokratietheorien sind trotz dieser Aufzählung immer selbstkritisch, weil die eigene Perspektive auf gesellschaftliche Ereignisse reflektiert wird, um sicher zu gehen, dass die eigenen Beiträge keine exkludierende Wirkung haben. Gleichzeitig sind sie selbstbewusst, weil sie sich von antidemokratischen Kräften nicht verdrängen oder davon abhalten lassen, kritische Befragung, Hinterfragung, Infragestellung wissenschaftlich zu betreiben und gesellschaftspolitisch zu unterstützen. Dabei sind u.a. folgende Fragen von Relevanz: Was ist unter zivilem Ungehorsam in Zeiten von Corona zu verstehen? Wie viel Konflikt braucht die Gesellschaft während einer Pandemie und ist Kritik illegitim, wenn die weit überwiegende Mehrheit der Menschen die Corona-Maßnahmen akzeptiert? Für Jonas Heller ist klar, dass Mittel umso kritischer betrachtet werden müssen, je anerkannter deren Zwecke sind (vgl. Heller 2020). Darin ist ihm zuzustimmen. Gegen Gesetze, Verordnungen und Verwaltungsakte, die demokratische Rechte unverhältnismäßig einschränken, helfen politische Initiativen, der Rechtsweg, Wahlen und sonstige Beteiligung am Diskurs. Die CoronaSituation ist zwar dynamisch, das ist Demokratie aber auch. Es gilt also wachsam zu sein, kritisch zu fragen, Antworten zu finden, demokratisch zu bleiben. Nur so können wir Mundschutz tragen, ohne mundtot zu sein.

\section{Literaturverzeichnis}

Abbas, Nabila. 2019. Jacques Rancière. In: Dagmar Comtesse et al. (Hrsg.), Radikale Demokratietheorie. Ein Handbuch. Berlin: Suhrkamp, 388-399.

Corona Monitor. Gesellschaftliche Transformation in Zeiten von Corona. https://coron amonitor.noblogs.org/. 
Fangerau, Heiner/Labisch, Alfons. 2020. Pest und Corona. Pandemien in Geschichte, Gegenwart und Zukunft. Freiburg: Herder Verlag.

Flügel-Martinsen, Oliver. 2019. Kritik. In: Dagmar Comtesse et al. (Hrsg.), Radikale Demokratietheorie. Ein Handbuch, 576-582.

Gramm, Christoph/Wittenberg, Mareike. 2018. Vorbemerkungen zu Art. 115a ff. GG. In: Hans Hofmann et al. (Hrsg.), GG: Kommentar zum Grundgesetz. Köln: Carl Heymanns Verlag, 1-75.

Heller, Jonas. 2020. Ausnahmezustand. In: Katharina Hoppe et al. (Hrsg.), Kritische Theorien in der Pandemie. Ein Glossar zur Corona-Krise. https://www.youtube.com /watch?v=Iktid9mD9rA. 02.06.2020.

Katzenberger, Paul. 2020. Corona und Demokratie: ,In der Krise rücken Staat und Bürger zusammen'. Süddeutsche Zeitung. www.sz.de/1.4871109. 02.06.2020.

Lemke, Matthias. 2017. Was heißt Ausnahmezustand? In: Matthias Lemke (Hrsg.), Ausnabmezustand: Theoriegeschichte - Anwendungen - Perspektiven. Wiesbaden: Springer VS, 1-12.

Leonhardt, Christian. 2017. Zwei Namen des Ausnahmezustandes. Giorgio Agamben und Jacques Rancière im Unvernehmen. In: Matthias Lemke (Hrsg.), Ausnahmezustand: Theoriegeschichte - Anwendungen - Perspektiven. Wiesbaden: Springer VS, 41-56.

Molly, Max. 2017. Differenz und Einheit. Zur Skizze eines demokratischen Ausnahmezustands nach und mit Otto Kirchheimer. In: Matthias Lemke (Hrsg.), Ausnahmezustand: Theoriegeschichte - Anwendungen - Perspektiven. Wiesbaden: Springer VS, 71-85.

Neft, Anselm. 2020. Von bösen Mächten wunderbar geborgen - Glaube an Verschwörungstheorien. ZEIT. https://www.zeit.de/kultur/2020-05/glaube-an-versch woerungstheorien-coronavirus. 02.06.2020.

Sabrow, Martin. 2020. Geschichte im Ausnahmezustand. Vier Thesen über Corona und die gesellschaftspolitischen Folgen. www.bpb.de/308316. 20.06.2020.

Sack, Detlef. 2019. Entscheidung. In: Dagmar Comtesse et al. (Hrsg.), Radikale Demokratietheorie. Ein Handbuch. Berlin: Suhrkamp, 504-512.

Schmid, Thomas. 2020. Virus und Ausnahmezustand - Was mit der Welt geschieht. WELT. https://www.welt.de/debatte/kommentare/article206678715/Cor onavirus-und-Ausnahmezustand-Was-mit-der-Welt-geschieht.html. 29.09.2020

Schmitt, Carl. 2015. Politische Theologie. Vier Kapitel zur Lehre von der Souveränität. Berlin: Duncker \& Humboldt.

Tagesschau. 2020. Ausnabmezustand als neue Normalität. https:/www.tagesschau.de/ faktenfinder/hintergrund/corona-chronik-pandemie-109.html. 29.09.2020.

Vasilache, Andreas. 2019. Dissens/Konflikt/Kampf. In: Dagmar Comtesse et al. (Hrsg.), Radikale Demokratietheorie. Ein Handbuch. Berlin: Suhrkamp, 492-503.

Winter, Yves. 2020. Democracy. In: Katharina Hoppe et al. (Hrsg.), Kritische Theorien in der Pandemie. Ein Glossar zur Corona-Krise. https://www.youtube.com/watc $\mathrm{h}$ ? $\mathrm{v}=$ T8bve5s4Gb4. 02.06.2020. 
